\title{
Recommendations for Routine Reporting on Indications for Cesarean Delivery in Developing Countries
}

Cynthia Stanton, $\mathrm{MPH}, \mathrm{PhD}^{1}$, Carine Ronsmans, MD, $\mathrm{DrPH}^{2}$, the *Baltimore Group on Cesarean

${ }^{1}$ Cynthia Stanton is an Assistant Professor in the Department of Population, Family and Reproductive Health at the Johns Hopkins Bloomberg School of Public Health, Baltimore, Maryland, USA ;

${ }^{2}$ Carine Ronsmans is a Reader in the Infectious Disease Epidemiology Unit at the London School of Hygiene and Tropical Medicine, London, United Kingdom; and *Members of the Baltimore Group on Cesarean are listed in Appendix 1.

Address correspondence to Cynthia Stanton, MPH, PhD, 118 Larch Road, Cambridge, MA 02138, USA

\section{KEYWORDS}

cesarean • classification • routine health information system

ABSTRACT: Background: Cesarean delivery rates are increasing rapidly in many developing countries, particularly among wealthy women. Poor women have lower rates, often so low that they do not reach the minimum rate of 1 percent. Little data are available on clinical indications for cesarean section, information that could assist in understanding why cesarean delivery rates have changed. This paper presents recommendations for routine reporting on indications for cesarean delivery in developing countries. These recommendations resulted from an international consultation of researchers held in February 2006 to promote the collection of comparable data to understand change in, or composition of, the cesarean delivery rate in developing countries. Methods: Data are presented from selected countries, categorizing cesareans by three classification systems. Results: A single classification system was recommended for use in both high and low cesarean delivery rate settings, given that underuse and overuse of cesarean section are evident within many populations. The group recommended a hierarchical categorization, prioritizing cesareans performed for absolute maternal indications. Categorization among the remaining nonabsolute indications is based on the primary indication for the procedure and include maternal and fetal indications and psychosocial indications, required for high cesarean delivery rate settings. Conclusions: Data on indications for cesarean sections are available everywhere the procedure is performed. All that is required is compilation and review at facility and at higher levels. Advocacy within ministries of health and medical professional organizations is required to advance these recommendations since researchers have inadequately communicated the health effects of both underuse and overuse of cesarean delivery.

Cesarean delivery rates are rising in many developing countries (1). The cesarean delivery rate for developing countries in 2002 was recently reported at 14 percent, with some Latin American and Asian countries reporting rates between 30 and 40 percent (2). Furthermore, some extreme socioeconomic 
disparities exist in access to cesarean delivery even in countries with reasonable national rates; women in the wealthiest households have rates well above 20 percent. At the same time, in poor countries and among the poorest households in many countries, cesarean delivery rates are so low that it is likely that women are dying from lack of access to the procedure. In 20 of 42 countries with available data, the cesarean delivery rate among the poorest quintile of households was less than 1 percent (3). The extent to which overuse of cesarean sections among certain segments of the population affects a health care system's ability to provide life-saving cesareans for poor women is unknown.

High and rising national rates indicate cause for concern but provide no information on why or how these rates are changing. Likewise, very low stagnant rates, as seen in much of sub-Saharan Africa, provide no assurance that the small number of cesarean sections are being conducted on the women in greatest need.

Developed countries historically have used data on medical indications for cesarean delivery to understand retrospectively the dramatic increases seen in its use (4-6). In general, both an expansion of the medical indications for its use $(7,8)$ and a liberalization of the threshold for these indications appear to have occurred over time (9). In response to these changes and also to the rise in nonmedically indicated cesareans, various means of classifying cesareans were developed. For example, the International Classification of Diseases (ICD) revisions 9 and 10 include mutually exclusive and hierarchical codes for repeat cesarean delivery (with no ability to identify the indication for repeated cesareans), breech presentation, dystocia, fetal distress, and other indications (10).

The Robson classification does not rely on medical indications at all but instead focuses on characteristics of who gave birth by cesarean delivery. This classification system uses 10 well-defined mutually exclusive groups to identify clinically relevant groups prospectively based on the following characteristics: parity (nulliparous vs multiparous), previous obstetric record of the woman, the course of labor and delivery, and gestational age (11). The 10 mutually exclusive groups are as follows: 1) nulliparous, single cephalic, greater than or equal to 37 weeks' gestation, spontaneous labor; 2) nulliparous, single cephalic, greater than or equal to 37 weeks' gestation, induced labor or cesarean before labor; 3) multiparous, single cephalic, greater than or equal to 37 weeks' gestation, no uterine scar, spontaneous labor; 4) multiparous, single cephalic, greater than or equal to 37 weeks' gestation, no uterine scar, induced labor or cesarean before labor; 5) multiparous, single cephalic, greater than or equal to 37 weeks' gestation, with uterine scar; 6) nulliparous singleton breech; 7) multiparous singleton breech, including previous scar; 8) multiple pregnancies (includes previous uterine scar); 9) singleton transverse, oblique, or unstable lie (including previous uterine scar); and 10) singleton cephalic, lesser than or equal to 36 weeks' gestation, including previous uterine scar. Still other classification 
systems categorize cesarean deliveries by their degree of urgency, with the objective of distinguishing between cesareans performed for life-threatening conditions to the mother, the fetus, or both, and cesareans performed for maternal or fetal compromise (12).

\section{Methods}

The need for comparable data to understand change in or composition of the cesarean birth rates in developing country settings was the impetus for an international meeting of researchers and obstetricians in February 2006. The decision to convene this meeting followed three failed attempts to gather and assess relevant developing country data on indications for cesarean delivery. These attempts included a literature review; direct requests to routine health information systems; and communication with professional contacts, requesting them to locate existing data on indications for cesarean delivery.

The results of the literature review concerning trends and regional variation in indication for cesarean delivery were inconclusive. The literature often included incomplete distributions of indications for cesarean deliveries, focused on selected populations (primiparous or singleton births), allowed for multiple indications for a cesarean delivery, and lacked comparable definitions. The direct requests to routine health information systems generated no responses, and communication to professional contacts in 26 countries generated data on indications from only one country (Mexico).

Given the paucity of accessible data, the meeting was planned with the following objectives: to share data and lessons from developing and selected developed countries about reporting on indications for cesarean deliveries and to propose recommendations for classifying indications for cesarean deliveries in routine health information systems in developing countries. Participants were invited based on their current involvement in cesarean-related research and to ensure geographic representation.

The meeting was cosponsored by the Initiative for Maternal Mortality Programme Assessment (IMMPACT) and the International Federation of Gynecology and Obstetrics and was held at the Johns Hopkins Bloomberg School of Public Health in Baltimore, Maryland. Participants from 10 countries, presenting data from 21 countries, attended the meeting. In this paper, selected examples of cesarean delivery classification systems presented during the meeting are highlighted and new recommendations for routine reporting on indications for cesarean deliveries proposed.

\section{Results}

Three different types of classification system were described: 1) a system based on a series of primary, mutually exclusive clinical indications; 2 ) a system to identify the degree of urgency or absolute need for cesarean delivery; and 3) the 
Robson classification. We present selected results from each of these classifications.

\section{Cesareans by mutually exclusive clinical indications}

Data on cesarean deliveries were presented from 42 hospitals in six Latin

American countries that use a standardized perinatal clinical record with mutually exclusive clinical indications (Argentina, Brazil, Colombia, Cuba, Guatemala, and Mexico) (13), and from the National Sentinel Caesarean Section Audit in England, Wales, and Northern Ireland (14). Hospital-based cesarean delivery rates across the six Latin American countries varied from 20 percent in Argentina to 36 percent in Brazil. Institutional delivery rates in these countries are between 85 and 100 percent in all, but 42 percent in Guatemala in 1999 (15). The leading indications for cesarean delivery include previous cesarean section, dystocia, fetal distress, and breech presentation, representing between 55 and 76 percent of all cesarean deliveries (Table 1). In these Latin American countries, emergencies represent only 6 percent or less of cesarean deliveries, whereas between 4 and 12 percent of cesareans are attributable to maternal disease. However, the distribution of indications is similar across countries and does not explain why or how rates in Brazil are nearly double those in Argentina.

Table 1. Percent Distribution of Mutually Exclusive Clinical Indications for Cesarean Delivery from 42 Hospitals in Six Latin American Countries, and Hospital-Based Cesarean Delivery Rates, 19982000

\begin{tabular}{lllllll} 
& $\begin{array}{c}\text { Argentina } \\
\text { (\%) }\end{array}$ & $\begin{array}{c}\text { Guatemala } \\
\text { (\%) }\end{array}$ & $\begin{array}{c}\text { Cuba } \\
\text { (\%) }\end{array}$ & $\begin{array}{c}\text { Mexico } \\
\text { (\%) }\end{array}$ & $\begin{array}{c}\text { Colombia } \\
\text { (\%) }\end{array}$ & $\begin{array}{c}\text { Brazil } \\
\text { (\%) }\end{array}$ \\
\hline $\begin{array}{l}\text { Previous } \\
\text { cesarean }\end{array}$ & 36.1 & 42.7 & 29.0 & 15.0 & 17.2 & 20.2 \\
section & & & & & & \\
Dystocia & 20.8 & 16.2 & 23.8 & 32.3 & 21.5 & 15.7 \\
Fetal distress & 8.4 & 11.9 & 22.8 & 15.0 & 19.6 & 19.3 \\
Breech & 9.9 & 16.6 & 10.0 & 8.5 & 11.1 & 8.6 \\
Maternal & 8.4 & 3.6 & 6.2 & 6.3 & 11.7 & 12.4 \\
Emergency & 6.4 & 3.2 & 2.8 & 3.1 & 5.9 & 5.0 \\
Other & 9.9 & 5.5 & 5.9 & 19.7 & 13.0 & 18.8 \\
Total & 100.0 & 100.0 & 100.0 & 100.0 & 100.0 & 100.0 \\
No. deliveries & 30,394 & 9,256 & 7,739 & 14,829 & 19,748 & 11,692 \\
Cesarean & 20.2 & 25.3 & 29.0 & 31.9 & 32.7 & 36.2 \\
delivery rates & & & & & &
\end{tabular}

Mutually exclusive clinical indications for cesarean delivery in England and Wales, and Northern Ireland are presented in Table 2. These data include more than 150,000 deliveries from 216 hospitals in a 3-month period in 2000 to 2001 
(14). The overall cesarean delivery rate was 22 percent in England and Wales and 24 percent in Northern Ireland. Leading indications include fetal compromise ( $22 \%$ and $14 \%$, respectively), failure to progress (20\% and $22 \%$, respectively), previous cesarean section (14\% and $24 \%$, respectively), and breech presentation (11\% and $10 \%$, respectively). Maternal request was the primary indication for 9 and 8 percent of cesarean deliveries, respectively. The sizable "other" category (representing 19\% and 18\%, respectively) included a wide range of maternal and fetal indications, for example, preeclampsia, eclampsia, or hemolysis-elevated liver enzymes-low platelet count (HELLP) syndrome; malpresentation; multiple pregnancy; and previous physical or emotional trauma during vaginal delivery among others.

Table 2. Percent Distribution of Mutually Exclusive Clinical Indications for Cesarean Delivery from 216 Hospitals in England and Wales, and Northern Ireland, and Hospital-Based Cesarean Delivery Rates, 2000-2001

Indication

Breech

Fetal compromise

Failure to progress

Antepartum hemorrhage

Previous cesarean section

Maternal request

Other

Total

Cesarean delivery rates

\section{England and Wales (\%) Northern Ireland (\%)}

10.8

22.0

20.4

5.1

13.8

9.0

18.9

100.0

21.5
9.6

14.3

22

3.9

23.9

8.2

18.1

100.0

23.9

\section{Classification by degree of urgency or absoluteness of the cesarean}

In addition to reporting on the primary indication for cesarean delivery, the National Sentinel Caesarean Section Audit in England, Wales, and Northern Ireland also classifies cesarean by degree of urgency, using the following categories: 1) immediate threat to the life of the woman or the fetus, 2) maternal or fetal compromise (not immediately life threatening), 3) no maternal or fetal compromise but need for early delivery, and 4) delivery timed to suit the mother and staff (14). Sixteen percent of procedures were performed because "an immediate threat to the life of the mother or fetus" existed (Fig. 1). The immediate threat category varied from 14 to 19 percent among regions. Thirty-two percent of procedures were performed for maternal or fetal compromise that was not immediately life threatening (range 30\%-34\%), 18 percent were performed because the mother needed early surgery without maternal or fetal compromise (range 17\%-22\%), and 31 percent were timed to suit the mother and staff (range $28 \%-34 \%)$. 


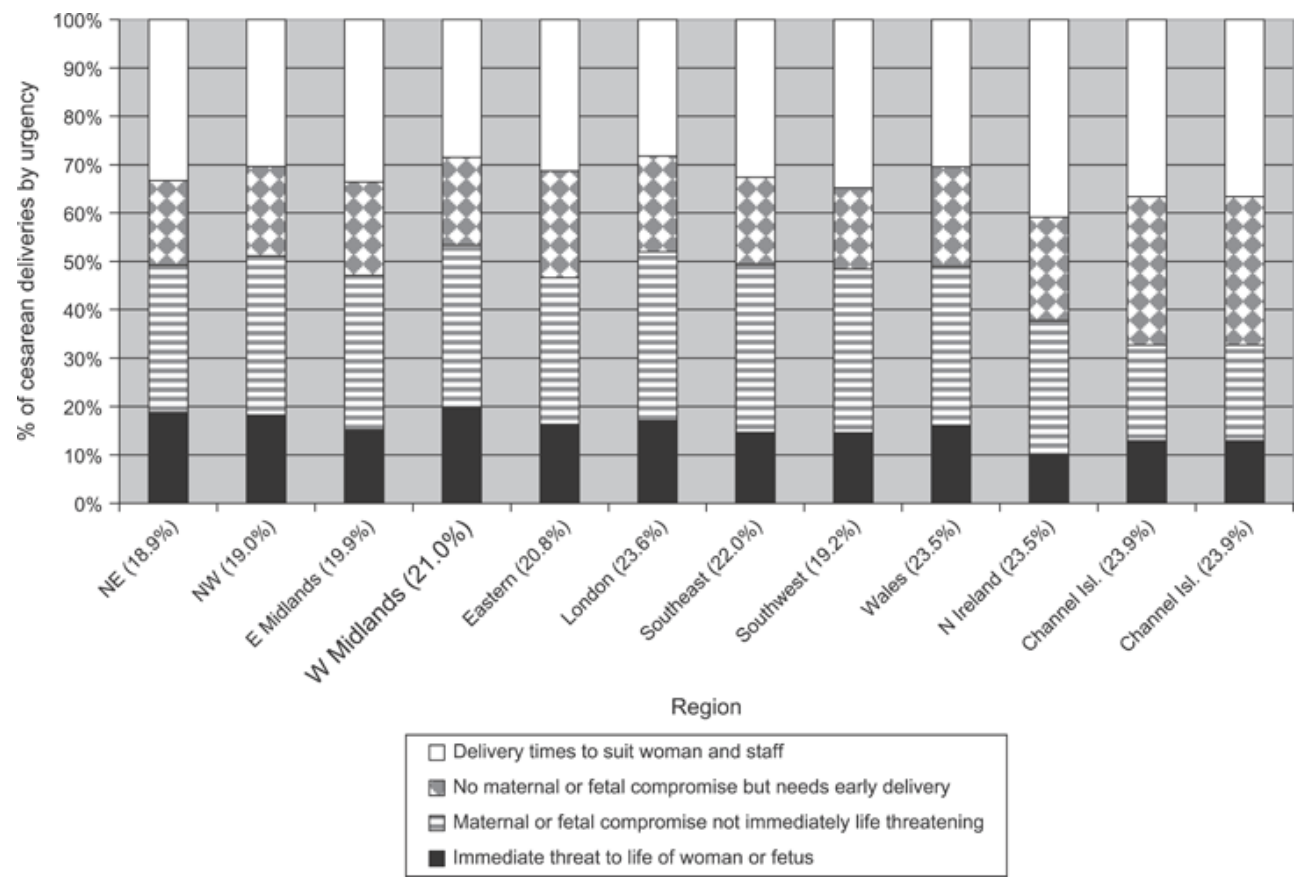

Fig. 1. Cesarean deliveries in England and Wales, and Northern Ireland, classified by region and by four categories of urgency, 2000-2001.

Countries participating in the Unmet Obstetric Need Network use a very different approach for documenting indications for cesarean delivery. Their aim is to identify major obstetric interventions performed to save a woman's life by identifying indications that are "absolute," that is, conditions that are thought to have a high probability of being fatal should the woman fail to obtain a major obstetric intervention. In 80 to 98 percent of women, the intervention is a cesarean section. Absolute maternal indications include severe antepartum hemorrhage due to placenta previa or placental abruption, unremitting postpartum hemorrhage, major cephalopelvic disproportion (including prerupture and rupture of the uterus), transverse lie, and brow presentation (16). Cesarean delivery data from four West African countries and Haiti were presented. All these countries have exceedingly low cesarean delivery rates (2\% or less), and rates of institutional birth range from 18 percent in Haiti and Niger to 78 percent in Benin (15). As shown in Fig. 2, absolute maternal indications are by far the leading indication for cesarean section in this sample of countries, with one-half to two-thirds of cesareans performed to save the life of the woman. Among absolute maternal indications, cephalopelvic disproportion predominates, followed by antepartum hemorrhage and face or brow presentation. Previous cesarean delivery, dystocia (other than those for absolute maternal indication), and fetal distress account for 19 to 35 percent of cesareans in these countries. 


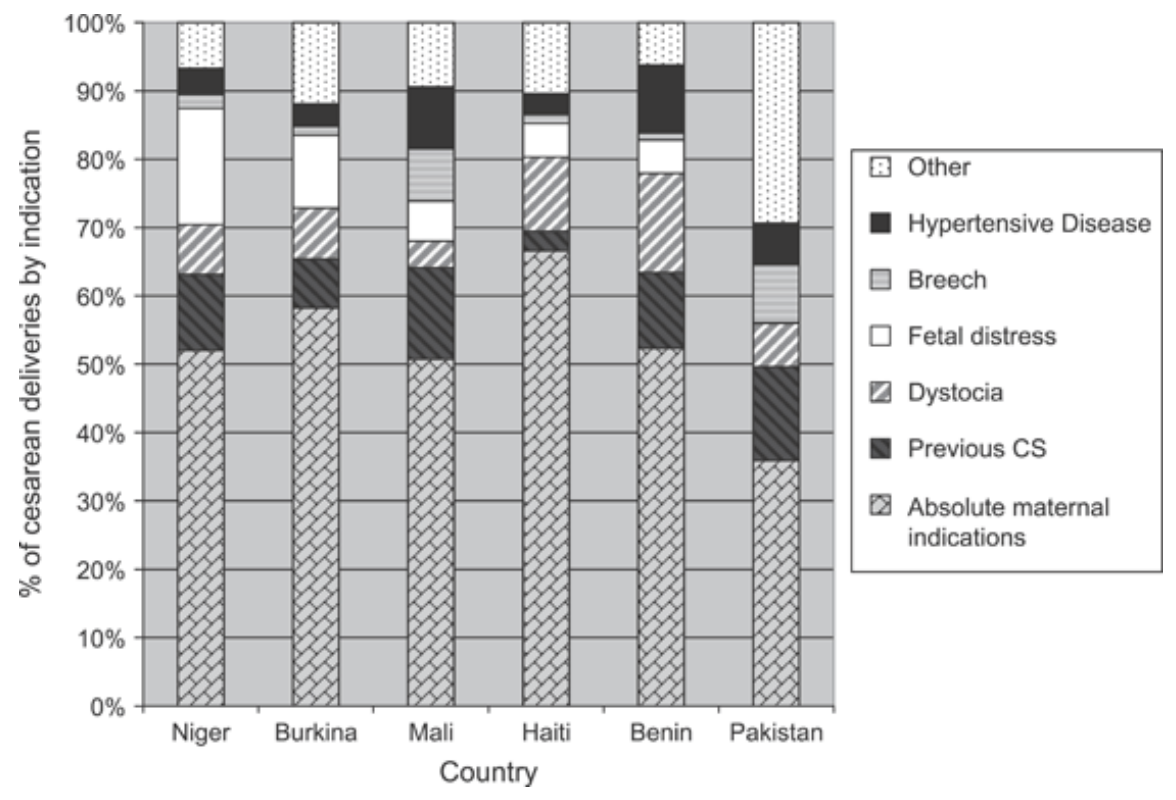

Fig. 2. Cesarean deliveries categorized by absolute maternal versus other indications from the Unmet Obstetric Need Network in Niger (1998), Burkina (1998-1999), Mali (1998), Haiti (1998), Benin (1999-2000), and Pakistan (1998-1999).

Another approach to focusing on the urgency of cesarean delivery was presented from Senegal, where the overall cesarean rate is 1.6 percent, and 64 percent of births occur in a health facility (15). The system used in Senegal to classify cesarean delivery is designed to capture both the urgency of and indication for the procedure. Three categories are defined as follows: 1) obligatory cesarean section where vaginal delivery is not possible and the absence of this procedure will lead to maternal or fetal death or other serious adverse outcome; 2) prudent cesarean section where vaginal delivery is theoretically possible but a cesarean section improves the mother's or baby's prognosis, and the indications include scarred uterus, breech presentation, fetal distress, "precious child"; and 3) necessary cesarean section, which is performed as a last recourse and results from poor management of labor within the facility or delayed referral. The original goal of the Senegalese Ministry of Health was to increase the cesarean delivery rate to 3 percent in the short term and to 10 percent in the long term, and to do so by favoring obligatory cesarean sections, controlling prudent cesareans, and reducing necessary cesareans.

Senegalese data on trends in cesarean delivery from 1992 to 2001 are shown in Fig. 3. Between 1992 and 1996, obligatory cesarean deliveries decreased from 50 to 44 percent and then stabilized through 2001; prudent cesareans remained stable around 30 percent over the 10-year period, and necessary cesareans increased from 22 to 27 percent. In addition to surveillance of cesarean delivery by category of urgency, cesarean-associated maternal deaths are also monitored. The overall percentage of maternal deaths among cesarean deliveries showed an impressive decrease from 4.7 percent in 1992 to 1.4 
percent in 2001; however, maternal mortality among the necessary cesarean deliveries remains high, decreasing from 7.8 to 6.4 percent between 2002 and 2001 (data not shown).

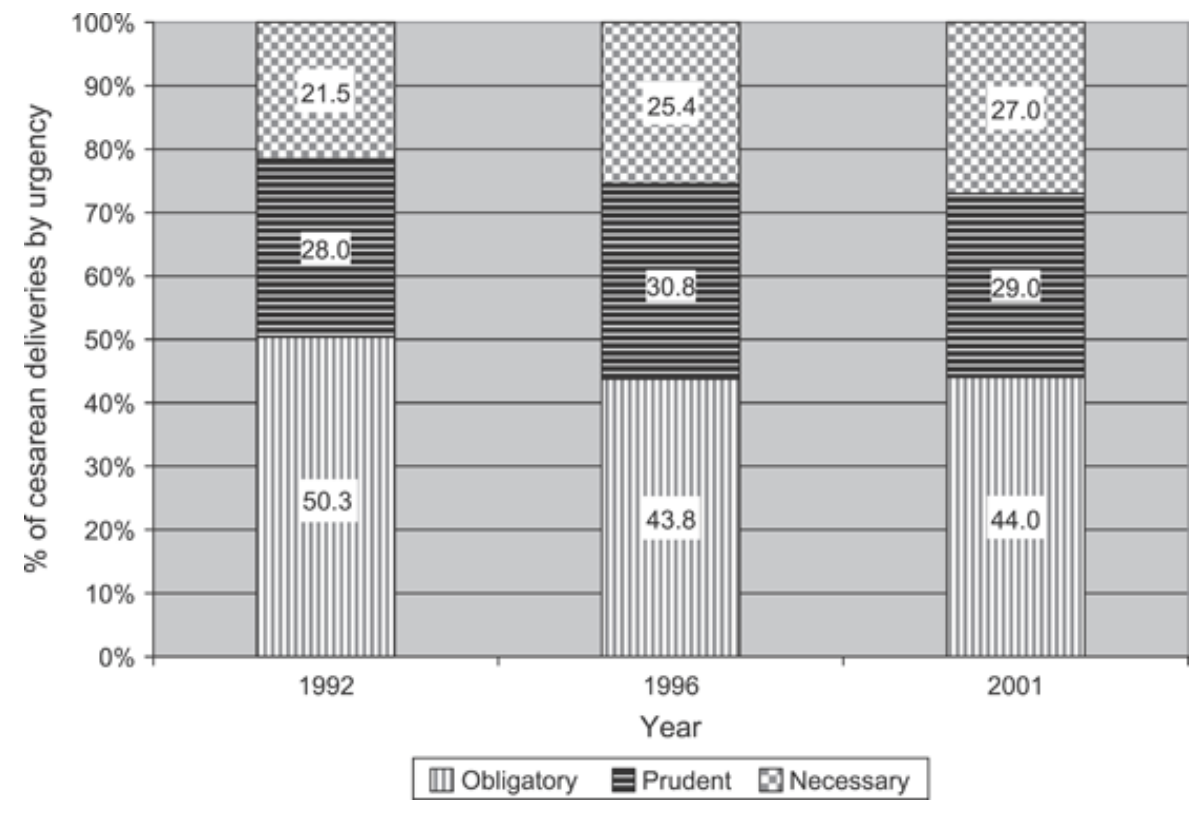

Fig. 3. Percent distribution of cesarean deliveries by urgency for the procedure, Senegal (19922001).

\section{Robson classification}

Cesarean delivery data categorized using the Robson classification were presented from South Africa. These data excluded referred patients and were collected from Kalafong Hospital in southwest Tshwane District between March and December 2005. The results were compared with those from the National Maternity Hospital in Dublin, Ireland, using the Robson classification to identify groups of women with excessive cesarean delivery rates and to make management changes to reduce cesarean deliveries in such groups (Table 3). In general, cesarean delivery rates in Kalafong are substantially higher than those at the Dublin National Maternity Hospital in 5 of the 10 Robson categories. Of particular concern are cesarean deliveries among mature single cephalic births with a spontaneous labor (groups 1 and 3). Both these groups represent large numbers of women; thus, even small changes in the cesarean delivery rate in these groups will result in a substantial increase in the overall rate. Moreover, increases in nulliparous women (group 1) will ultimately lead to increases in cesareans among women with a uterine scar (group 5). These findings suggested to surveillance staff that improved diagnosis and routine management of labor were needed. The very high cesarean delivery rate in Kalafong in group 5 appears to be associated with the inability to offer women adequate pain relief and proper monitoring during labor to enable a woman to attempt a vaginal birth after a previous cesarean section. The very low cesarean delivery rate in group 9 
(singleton transverse, oblique, or unstable lie) is believed to be due to poor obstetric skills in assessing the lie of the fetus.

Table 3. Cesarean Delivery Rates by Clinical Indication using the Robson Classification * from the National Maternity Hospital in Dublin, Ireland (2002), and from Kalafong Hospital, Southwest Tshwane, South Africa (2005)

\begin{tabular}{llll}
$\begin{array}{l}\text { Robson } \\
\text { Group }\end{array}$ & \multicolumn{1}{c}{$\begin{array}{c}\text { National Maternity } \\
\text { Hospital (Dublin) }\end{array}$} & $\begin{array}{c}\text { Kalafong } \\
\text { Hospital }\end{array}$ & $\begin{array}{c}\text { Number of Women at the } \\
\text { Kalafong Hospital }\end{array}$ \\
\hline 1 & 7.3 & 15.1 & 1,592 \\
2 & 24.5 & 48.4 & 192 \\
3 & 1.1 & 8.3 & 2,060 \\
4 & 4.6 & 44.2 & 224 \\
5 & 50.4 & 85.2 & 298 \\
6 & 88.9 & 68.0 & 50 \\
7 & 70.9 & 64.6 & 82 \\
8 & 58.9 & 53.9 & 52 \\
9 & 100.0 & 30.0 & 20 \\
10 & 26.9 & 29.3 & 805
\end{tabular}

* See text for description of the Robson classification.

\section{Recommendations for routine reporting on cesarean delivery}

After country presentations at the meeting, participants were divided into working groups focusing on either high or low cesarean delivery rate settings. Groups were asked to propose recommendations appropriate for routine reporting on indications for cesarean delivery in developing countries, with the goal of ensuring reliable data to permit review of the indications for cesarean delivery and to allow for site-specific decision making to address key areas of concern.

The results of these discussions were similar in the two groups, which led to a consensus that one set of recommendations was appropriate in recognition of the fact that groups of women are present within the same population with very high and very low cesarean section rates. Most of the discussion leading up to the final recommendations focused on the criteria used to identify major antepartum hemorrhage and obstructed labor. The recommended categorization reflected the following principles: first, it is essential to document that some cesareans are being performed to save the life of the woman; second, it is essential that a classification system provides categories that capture the reality of very high cesarean delivery settings (e.g., psychosocial considerations such as maternal request); and third, it is essential that a classification system provides data on the balance between cesarean deliveries for maternal versus 
fetal compromise. The recommended classification is therefore divided into absolute, maternal, and nonabsolute indications. The absolute maternal indications include obstructed labor (including severe deformed pelvis, failed trial of labor), major antepartum hemorrhage and grade 3 or 4 placenta previa, malpresentation (including transverse, oblique, and brow), and uterine rupture. The nonabsolute indications include failure to progress in labor, including prolonged labor; failed induction; previous cesarean delivery; genitourinary fistula or third-degree tear repair; antepartum hemorrhage, excluding those for absolute indications and including abruptio placentae; maternal medical diseases; severe preeclampsia or eclampsia; psychosocial indications, including maternal request, "precious" pregnancy; fetal compromise, including fetal distress, cord prolapse, severe intrauterine growth retardation; and breech presentation.

The proposed classification is hierarchical in that cesarean deliveries are first categorized by whether the procedure was performed for absolute maternal indication, which includes obstructed labor, major antepartum hemorrhage (including grades 3 and 4 placenta previa), malpresentation (defined as transverse, oblique, and brow presentation), and uterine rupture. The nonabsolute indications are not hierarchical and represent the primary indication for cesarean. These indications include previous cesarean section, failure to progress (prolonged labor, failed induction), genitourinary fistula or third-degree tears, antepartum hemorrhage (excluding the absolute causes of antepartum hemorrhage), maternal medical diseases, psychosocial factors, fetal compromise, and breech presentation.

\section{Discussion}

It is an accepted fact that indications for cesarean deliveries are recorded in surgical registers; individual medical records; and, occasionally, delivery room logbooks in all facilities that perform cesarean deliveries. The results of our exercise to compile these data, however, suggest that they are seldom aggregated or passed on to higher levels for review on a routine basis. Meanwhile, cesarean delivery rates rise in many countries or within certain segments of populations and continue to rise with little or no notice and even less understanding of why these cesareans are being performed. More tragic is the case of very low cesarean delivery settings, where national rates may suggest provision of a higher level of life-saving care than is reality due to the unequal access to cesarean delivery among women giving birth.

The data presented by meeting participants attest to the lack of standardization concerning how and why existing data are collected. For example, the priority for data collection in Senegal, and also in the countries in the Unmet Obstetric Need Network, is clearly as a means of reducing maternal mortality. However, this focus on urgency is not restricted to high mortality settings-the United Kingdom also uses an urgency-based classification system. In contrast, in South Africa, referral patients were excluded from the analysis and a conscious focus was made to identify cesareans conducted in excess of medical need, which was 
determined by means of comparison with data from Ireland. Motivation for the analysis in the Latin American countries was also to explain overuse of cesarean delivery and relied on cross-country comparisons at one point in time. However, a comparison of mutually exclusive indications provided few insights into the high but varying rates when compared across different countries. It is also noteworthy that none of the participants presented data classified by ICD codes

\section{Conclusions}

The recommendations proposed by this group, which first distinguish cesarean deliveries performed for a series of absolute maternal indications and then specify those performed for nonabsolute indications that include fetal and psychosocial indications, are not designed for prospective clinical decision making. They are proposed to address the complete dearth of data on indications for cesarean deliveries in the developing world. Thus, their purposes are to encourage and standardize the monitoring of cesarean deliveries in all settings, and are conceived as a tool to enable concurrent analysis and long-term data review that can be included in developing countries' routine health information systems. This achievement will involve adding standardized data items on cesarean delivery to routine annual reports. Given the burden of data reporting in most countries, it is advised that such data on indications for cesarean delivery be collected in registers or delivery logbooks, or by means of special data collection activities organized annually or biannually.

Two steps have been taken to encourage implementation of these recommendations: first, the International Federation of Gynecology and Obstetrics will consider endorsement of these recommendations later this year, and second, the recommendations will be referred to in the upcoming World Health Organization manual Handbook on Monitoring Availability and Use of Obstetric Care. Acceptance of this system will require advocacy by influential members of ministries of health and professional medical associations within countries. With the exception of Latin America and the U.K., most of the meeting participants commented that few people in their countries were interested in the data they presented at this meeting. In developing countries, researchers have very inadequately communicated the detrimental health effects of both underuse and overuse of cesarean deliveries $(17,18)$ and have not explored such effects on the health care system. Routine monitoring of indications for cesarean section would provide reliable data on which to base decisions about management of labor and delivery and allocation of resources in an effort to improve maternal and newborn outcomes. The data exist. They simply need to be compiled and examined in a systematic manner. 


\section{Appendix 1. Members of the Baltimore Group on Cesarean}

Patricia Bailey, Family Health International, Research Triangle Park, North Carolina, USA; Jose Belizán, Department of Mother and Child Health Research, Institute for Clinical Effectiveness and Health Policy, Buenos Aires, Argentina; Pierre Buekens, Tulane School of Public Health and Tropical Medicine, New Orleans, Louisiana, USA; Qian Chen, Peking University, First Hospital, Beijing, China; Cheikh T. Cisse, University of Dakar, Dakar, Senegal; Andrea A. Creanga, Johns Hopkins Bloomberg School of Public Health, Baltimore, Maryland, USA; Luc de Bernis, United Funds for Population, Africa Division, Addis-Ababa, Ethiopia; Dominique Dubourg, Institute for Tropical Medicine, Antwerp, Belgium; El Marie Farrell, Medical Research Council, University of Pretoria, Pretoria, South Africa; Sara A. Holtz, Johns Hopkins Bloomberg School of Public Health, Baltimore, Maryland, USA; Theo Lippeveld, John Snow International, Boston, Massachusetts, USA; Allisyn Moran, International Centre for Diarrhoeal Disease Research, Dhaka, Bangladesh; Dorothy Shaw, Departments of Obstetrics and Gynaecology and Medical Genetics, University of British Columbia, British Columbia's Women's Hospital and Health Centre Society, Vancouver, British Columbia, Canada; Tran Son Thach, Hung Vuong Hospital, Ho Chi Minh City, Vietnam; Jane Thomas, Former Director, National Collaborating Centre for Women's and Children's Health, Royal College of Obstetricians and Gynaecologists, London, United Kingdom, and Consultant Obstetrician and Gynecologist; Linda L. Wright, National Institutes of Child Health and Human Development, National Institutes of Health, Bethesda, Maryland, USA.

\section{References}

1. Stanton C, Holtz S. Levels and trends in cesarean birth in developing countries. Stud Fam Plann 2006;37(1):41-48.

2. Betran AP, Merialdi M, Lauer JA, et al. Rates of caesarean section: Analysis of global, regional and national estimates. Paediatr Perinat Epidemiol 2007;21(2):98-113.

3. Ronsmans C, Holtz S, Stanton C. Socioeconomic differentials in caesarean rates in developing countries: A retrospective analysis. Lancet 2006;368(9546):1516-1523.

4. Notzon FC, Cnattingius S, Bergsjo P, et al. Cesarean section delivery in the 1980s: International comparison by indication. Am J Obstet Gynecol 1994;170(2):495-504.

5. Anderson GM, Lomas J. Determinants of the increasing cesarean birth rate. Ontario data 1979 to 1982. N Engl J Med 1984;311(14):887-892. 
6. Bottoms SF, Rosen MG, Sokol RJ. The increase in the cesarean birth rate. $N$ Engl J Med 1980;302(10):559-563.

7. Penn Z, Ghaem-Maghami S. Indications for caesarean section. Best Pract Res Clin Obstet Gynaecol 2001;15(1):1-15.

8. Ganesh V, Apuzzio JJ, Nimmo RA, et al. Rates of cesarean section and perinatal outcome: Stillbirths. Eur J Obstet Gynecol Reprod Biol 1993;50(1):2531.

9. Leitch CR, Walker JJ. The rise in caesarean section rate: The same indications but a lower threshold. Br J Obstet Gynaecol 1998;105(6):621-626.

10. World Health Organization. International Classification of Diseases, 9th Revision. Geneva, Switzerland: Author, 1977.

11. Robson M. Classification of caesarean sections. Fetal Matern Med Rev 2001;12:23-39.

12. Lucas DN, Yentis SM, Kinsella SM, et al. Urgency of caesarean section: A new classification. J R Soc Med 2000;93(7):346-350.

13. Althabe F, Belizan JM, Villar J, et al. Mandatory second opinion to reduce rates of unnecessary caesarean sections in Latin America: A cluster randomised controlled trial. Lancet 2004;363(9425):1934-1940.

14. Thomas J, Paranjothy S, Royal College of Obstetricians and Gynaecologists Clinical Effectiveness Support Unit. The National Sentinel Caesarean Section Audit Report. London, UK: Royal College of Obstetricians and Gynaecologists Press, 2001.

15. ORC Macro. STATCompiler. Accessed on November 1, 2007. Available at: http://www.statcompiler.com/.

16. Unmet Obstetric Need Network. Tackling Unmet Obstetric Needs.

Comparative Study in Benin, Burkina Faso, Haiti, Mali, Morocco, Niger, Pakistan and Tanzania. Antwerp, Belgium: Institute of Tropical Medicine, 2002.

17. World Health Organization. World Health Report 2005: Make Every Mother and Child Count. Geneva, Switzerland: Author, 2005.

18. Villar J, Valladares E, Wojdyla D, et al. Caesarean delivery rates and pregnancy outcomes: The 2005 global survey on maternal and perinatal health in Latin America. Lancet 2006;367(9525):1819-1829. 